\title{
Peroxide Bond Driven Dissociation of Hydroperoxy-Cholesterol Esters Following Collision Induced Dissociation
}

\author{
Patrick M. Hutchins, Robert C. Murphy
}

Department of Pharmacology, MSC 8303, University of Colorado Denver, RC1 South, L18-6120, 12801 E. 17th Ave, P.O. Box 6511, Aurora, CO 80045, USA

\begin{abstract}
Oxidative modification of polyunsaturated fatty acids, which occurs through enzymatic and nonenzymatic processes, is typically initiated by the attachment of molecular oxygen to an unsaturated fatty acyl chain forming a lipid hydroperoxide $(\mathrm{LOOH})$. Enzymatic pathways are critical for cellular homeostasis but aberrant lipid peroxidation has been implicated in important pathologies. Analysis of primary oxidation products such as hydroperoxides has proven to be challenging for a variety of reasons. While negative ion electrospray ionization has been used for the specific detection of some $\mathrm{LOOH}$ species, hydroperoxide dehydration in the ion source has been a significant drawback. Here we describe positive ion electrospray ionization of ammoniated 13-hydroperoxy-9Z, 11E-octadecadienoyl cholesterol and 9-hydroperoxy-10E, 12Z-octadecadienoyl cholesterol, $\left[\mathrm{M}+\mathrm{NH}_{4}\right]^{+}$, following normal phase high-pressure liquidchromatography. Dehydration in the ion source was not prevalent and the ammoniated molecular ion was the major species observed. Collisionally induced dissociation of the two positional isomers yielded unique product ion spectra resulting from carbon-carbon cleavages along their acyl chains. Further investigation of this behavior revealed that complex collision induced dissociations were initiated by scission of the hydroperoxide bond that drove subsequent acyl chain cleavages. Interestingly, some of the product ions retained the ammonium nitrogen through the formation of covalent carbon-nitrogen or oxygen-nitrogen bonds. These studies were carried out using hydroperoxy-octadecadienoate cholesteryl esters as model compounds, however the observed mechanisms of $\left[\mathrm{LOOH}+\mathrm{NH}_{4}\right]^{+}$ionization and dissociation are likely applicable to the analysis of other lipid hydroperoxides and may serve as the basis for selective $\mathrm{LOOH}$ detection as well as aid in the identification of unknown lipid hydroperoxides.
\end{abstract}

Key words: 9-hydroperoxy-10E,12Z-octadecdienoyl cholesterol, 13-hydroperoxy-9Z,11 Eoctadecadienoyl cholesterol, Hydroperoxy cholesterol esters, Lipid hydroperoxides, peroxide scission, Collisional activation cholesterol esters, deuterium labeled cholesterol esters, N-15 ammonium adduct

Electronic supplementary material The online version of this article (doi:10.1007/s13361-011-0109-9) contains supplementary material, which is available to authorized users.

Correspondence to: Robert C. Murphy; e-mail: Robert.Murphy@ucdenver.edu

\section{Introduction}

The peroxidation of polyunsaturated fatty acids is a 1 ubiquitous transformation catalyzed by a large number of enzymes throughout the animal and plant kingdoms. In 
humans, peroxidation of fatty acids can be catalyzed by lipoxygenases, cyclooxygenases, cytochrome P-450s, as well as other enzymes [1-3]. For example, the enzymatic cascades that convert arachidonic acid to prostaglandins and leukotrienes have in common an initial peroxidation step where the first products are hydroperoxides that are converted into biologically active lipid mediators by subsequent enzymatic transformations. The initial lipid peroxides (LOOH) can also play important roles in a great variety of biological processes, including regulation of vascular tone, chemotaxis, endothelial permeability, gastric secretion, and more [4]. In addition to the enzymatic formation of $\mathrm{LOOH}$, nonenzymatic free radical processes can efficiently oxygenate polyunsaturated fatty acids and polyunsaturated acyl groups (esters) found in phospholipids, glyceryl lipids, and cholesteryl esters producing vast varieties of $\mathrm{LOOH}$ and their degradation products [5]. In many cases, nonenzymatic peroxidation products exert significant biological activity, including active oxidized phospholipids and cholesteryl esters [6,7]. Aberrant lipid oxidation, both enzymatic and otherwise, has been implicated in important human diseases such as atherosclerosis and neurodegenerative disorders $[8,9]$. Despite the biological importance of lipid peroxidation, the direct analysis of intact $\mathrm{LOOH}$ has remained an analytical challenge.

Several approaches have been taken for the analysis of fatty hydroperoxides. The most common methods employ high-pressure liquid-chromatography and UV detection [10]. These methods work well for species with sufficiently large UV-extinction coefficients but do not allow for selective detection of individual LOOH species. Gas-chromatography mass-spectrometry (GC-MS) provides detailed structural information for many oxidized lipid species, however, several studies have shown that polyunsaturated peroxide TMS ethers, especially allylic species, undergo extensive thermolysis at the injection port and cannot pass through a gas chromatograph [11]. Therefore, reduction of the hydroperoxide moiety followed by derivatization of the resultant carbinol has been required to yield suitable analytes for GC-MS analysis [12]. Qualitative and quantitative measurements of arachidonate and linoleate hydroperoxides, as free acids, have been reported using electrospray ionization tandem mass spectrometry (MS/MS) of the carboxylate anion $[13,14]$. An intact molecular anion $[\mathrm{M}-\mathrm{H}]^{-}$could be observed in these $\mathrm{LC} /$ MS and LC/MS/MS studies but facile dehydration inside the electrospray source resulted in significant conversion of hydroperoxide to an oxo moiety prior to introduction into the mass spectrometer. Further complicating these analyses was the fact that collision induced dissociation (CID) of the hydroperoxide molecular anion yielded the exact same CID mass spectrum as the corresponding ketone $[14,15]$. This could be problematic given the similar chromatographic properties of congruent hydroperoxide and oxo-fatty acids (e.g., retention times of 9hydroperoxyoctadecadienoate and 9-oxo-octadecadienoate by reverse phase separation).

Here we report positive ion electrospray MS/MS behavior of ammoniated 9-and 13-hydroperoxy-octadecadienoic
(HpODE) cholesteryl esters $\left[\mathrm{M}+\mathrm{NH}_{4}\right]^{+}$. Such molecules have been observed to accumulate in atherosclerotic plaque and, therefore, are interesting analytical targets [16]. These analyses required no prior derivatization and yielded abundant molecular ions. CID of the molecular ions yielded spectra that were unique for the two positional isomers. Further investigation into the mechanisms of CID revealed that hydroperoxide scission was the initiating step in the dissociation of the ammoniated molecular ion. Positive ion MS/MS of ammoniated LOOHs species could be the basis for the development of selective analyses as well as provide structural evidence for the identification of unknown lipid peroxides.

\section{Experimental}

\section{Materials}

The 9-hydroperoxy-10E,12Z-octadecdienoyl cholesterol (9HpODE-CE) and 13-hydroperoxy-9Z,11E-octadecadienoyl cholesterol (13-HpODE-CE) were obtained from Cayman Chemical (Ann Arbor, MI) as a mixture. The $[9,10,12,13$ ${ }^{2} \mathrm{H}_{4}$ ]-10Z,12Z-octadecadienoic acid $(>98 \%$ atom percent excess ${ }^{2} \mathrm{H}_{4}$, Cayman Chemical) was esterified to cholesterol to yield $\left[9,10,12,13-{ }^{2} \mathrm{H}_{4}\right]-10 \mathrm{Z}, 12 \mathrm{Z}$-octadecadienoyl cholesterol as previously described [17]. This deuterated cholesteryl ester was converted to $\left[9,10,12,13-{ }^{2} \mathrm{H}_{4}\right]-9$-hydroperoxy-10E,12Zoctadecdienoyl cholesterol $\left(\mathrm{D}_{4}-9-\mathrm{HpODE}-\mathrm{CE}\right)$ and $\left[9,10,12,13-{ }^{2} \mathrm{H}_{4}\right]$-13-hydroperoxy-9Z,11E-octadecadienoyl cholesterol ( $\left.\mathrm{D}_{4}-13-\mathrm{HpODE}-\mathrm{CE}\right)$, among other products, by radical peroxidation initiated with $10 \mathrm{mM} \mathrm{2,2'-azobis-2-}$ methyl-propanimidamide, dihydrochloride (Cayman Chemical) essentially following the procedure previously described [18]. The specific $\mathrm{D}_{4}-9-\mathrm{HpODE}-\mathrm{CE}$ and $\mathrm{D}_{4}-13-\mathrm{HpODE}-\mathrm{CE}$ isomers were separated by normal phase HPLC described below. The ${ }^{15} \mathrm{NH}_{4} \mathrm{Cl}$ was obtained from Sigma Aldrich (98 atom $\%{ }^{15} \mathrm{~N}$, St. Louis, MO).

\section{$N P-H P L C$}

Analytes were separated by normal phase chromatography on a $150 \times 2.1 \mathrm{~mm}, 5 \mu \mathrm{m}$, Acentis $\mathrm{Si}$ column (Supelco, Bellefonte, PA) using a gradient of methyl-tert-butyl ether (MTBE) in iso-octane going from $4.5 \%$ to $45 \%$ MTBE as previously reported [17, 19]. Ammonium adducts were formed in the electrospray ion source by the addition ( $\sim 10 \%$ of gradient flow rate) of $10 \mathrm{mM}$ ammonium acetate $\left(\mathrm{NH}_{4} \mathrm{OAc}\right)$ in $95: 5$ acetonitrile: $\mathrm{H}_{2} \mathrm{O}(\mathrm{vol} / \mathrm{vol})$ post-column.

\section{Mass Spectrometry}

Product ion spectra $\left(\mathrm{MS}^{2}\right.$ and $\left.\mathrm{MS}^{3}\right)$ were collected on an $\mathrm{AB}$ SCIEX 5500 triple quadrupole linear ion trap (LIT) hybrid mass spectrometer operated in positive ion mode. The electrospray voltage was set at $5500 \mathrm{~V}$, the declustering potential was $15 \mathrm{~V}$, the collision energy was 10 . For $\mathrm{MS}^{3}$ 
experiments excitation energy was 0.01 , all other parameters were the same as above. Precursor ion scanning experiments were carried out using an AB SCIEX (Foster City, CA) API 3200 triple quadrupole mass spectrometer operated in positive ion mode. Precursors of $\mathrm{m} / \mathrm{z} 369.3$ were detected by scanning the first quadrupole from $\mathrm{m} / z 650$ to 800 over $3 \mathrm{~s}$ intervals; all other parameters were the same. Highresolution accurate mass measurements were made using a Thermo Finnigan LTQ-FT Ultra linear ion trap Fourier transform ion cyclotron resonance hybrid mass spectrometer (San Jose, CA). For these experiments a solution of 9- and 13-HpODE-CE $(1 \mathrm{ng} / \mu \mathrm{L})$ in 9:1 $\mathrm{MeOH}: \mathrm{H}_{2} \mathrm{O}$ (vol/vol) containing ammonium acetate $(10 \mathrm{mM})$ was infused into the electrospray source at $20 \mu \mathrm{L} / \mathrm{min}$. Product ion spectra were collected from $m / z 250-710$, the electrospray voltage was $5000 \mathrm{~V}$, capillary temperature was $250{ }^{\circ} \mathrm{C}$, capillary voltage was $100 \mathrm{~V}$, ion isolation window was $15 \mathrm{u}$, and collision energy was $12 \%$. The large isolation window was necessary in order to obtain product ions in sufficient abundance for detection in the FTICR with the resolution parameter set at $10^{5}$. This unusually wide isolation window did not affect which ions were observed in the product ion spectra, or their relative intensities. The mass spectrometer was automatically calibrated using the manufacturer's recommended positive mode calibration solution containing L-methionyl-arginyl-phenylalanyl-alanine acetate, Ultramark 1621, and caffeine.

\section{Results}

\section{NP-HPLC-MS/MS Detection} of 9- and 13-HpODE-CE

Since the authentic standards of 9-HpODE-CE and 13HpODE-CE were only available as a mixture, chromatographic separation was critical for obtaining independent mass spectra. By normal phase HPLC (NP-HPLC), 9HpODE-CE and 13-HpODE-CE were well retained and completely separated (Figure 1a). The major ion species was the ammoniated molecular ion, $\left[\mathrm{M}+\mathrm{NH}_{4}\right]^{+} \mathrm{m} / z 698$ (>90\%). Water loss, $\left[\mathrm{M}-\mathrm{H}_{2} \mathrm{O}+\mathrm{NH}_{4}\right]^{+} \mathrm{m} / z$ 680, and water plus ammonia loss, $\left[\mathrm{M}-\mathrm{H}_{2} \mathrm{O}-\mathrm{NH}_{3}\right]^{+} m / z 663$, were also observed as minor ions (Figure 1a inset). The commercially available mixture was contaminated with small amounts of the all-trans isomers as well as the dehydration products, 9and 13-oxo-octadecadienoic cholesterol esters (9-oxo-ODE$\mathrm{CE}$ and 13-oxo-ODE-CE) (Figure 1a).

\section{Collision Induced Dissociation of 9 and 13-HpODE CE}

Each 9- and 13-HpODE-CE produced unique product ion spectra after collisional activation of the corresponding ammoniated molecular ion although there were a number of common ions (Figure 2a and b). Both spectra had the most abundant product ion at $\mathrm{m} / \mathrm{z} 369$, which corresponded

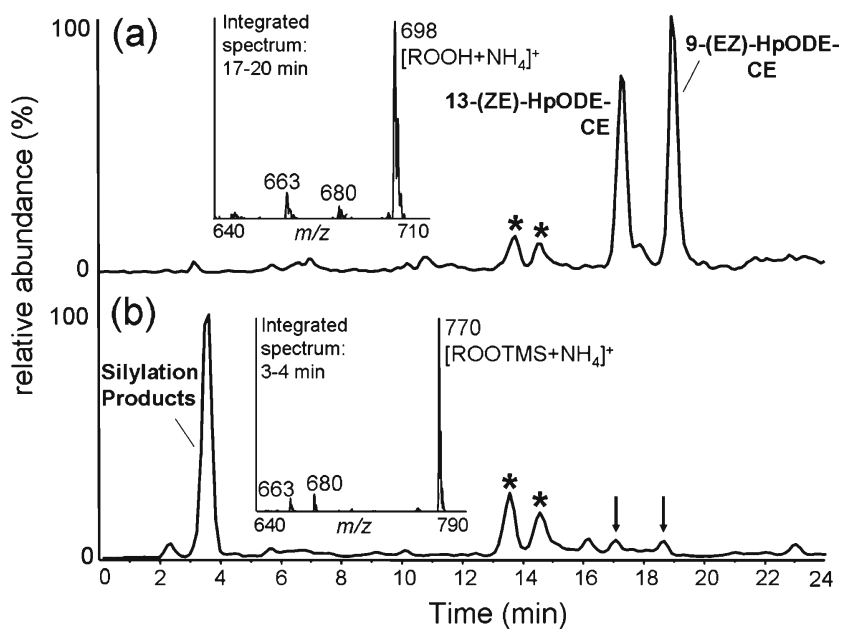

Figure 1. NP-HPLC separation of 9- and 13-HpODE-CEs before and after trimethylsilylation. Chromatograms are total positive ion currents of precursors of $\mathrm{m} / \mathrm{z} 369.3$ scanning $(\mathrm{m} / \mathrm{z}$ 650-800) of the starting material mixture (a) and the derivatization products (b). The asterisks indicate 9- and 13-oxo-ODE-CE impurities, the arrows indicate residual underivatized 9- and 13HpODE-CE. Insets: integrated mass spectra, averaged over the elution windows of both major peaks from $\mathrm{m} / \mathrm{z} 640$ to 710 or 790

to the dehydrated cholesterol core as previously reported [20]. Both spectra also contained several product ions consistent with neutral losses of $\mathrm{H}_{2} \mathrm{O}(\mathrm{m} / z 680), \mathrm{H}_{2} \mathrm{O}+\mathrm{NH}_{3}$ ( $m / z$ 663), and $\mathrm{H}_{2} \mathrm{O}_{2}+\mathrm{NH}_{3}(\mathrm{~m} / z$ 647). Both product ion spectra also showed minor ions at $m / z 295$ and 277, which were consistent with consecutive water losses from the free HpODE acyl component, [HpODE $\left.-\mathrm{H}_{2} \mathrm{O}+\mathrm{H}\right]^{+}, m / z$ 295 and $\left[\mathrm{HpODE}-2\left(\mathrm{H}_{2} \mathrm{O}\right)+\mathrm{H}\right]^{+}, m / z$ 277. Product ions unique for the individual isomers were observed in the $\mathrm{m} / \mathrm{z} 400$ 600 range. The 13-HpODE-CE species yielded product ions at $\mathrm{m} / \mathrm{z} 451,549,563$, and 580, while the 9-HpODE-CE product ion spectra contained unique ions at $\mathrm{m} / \mathrm{z} 491$ and 540 .

To determine whether these unique fragment ions retained the intact hydroperoxide moiety, the mixture was derivatized using the silylating reagent BSTFA and the products were subjected to NP-HPLC MS/MS (Figure 1b). The conversion to hydroperoxy TMS ethers was complete in less than $1 \mathrm{~min}$, and no side products or degradation products were observed. Although these compounds were poorly retained on NP-HPLC they were nonetheless slightly separated and thus, independent CID spectra could be obtained (Figure 2c and d). Interestingly, the CID spectra of the TMS ether derivatives were identical to those of the underivatized species except for the intact molecular adduct ion $\left[\mathrm{M}+\mathrm{NH}_{4}\right]^{+}$, which was $72 \mathrm{u}$ higher in mass than the underivatized hydroperoxide. These data revealed, first of all, that the hydroperoxy group was readily derivatized to the trimethylsilyl peroxyether, second, that none of the product ions retained the TMS group and, by extension, no products of the underivatized species retained the exchangeable peroxide hydrogen. 


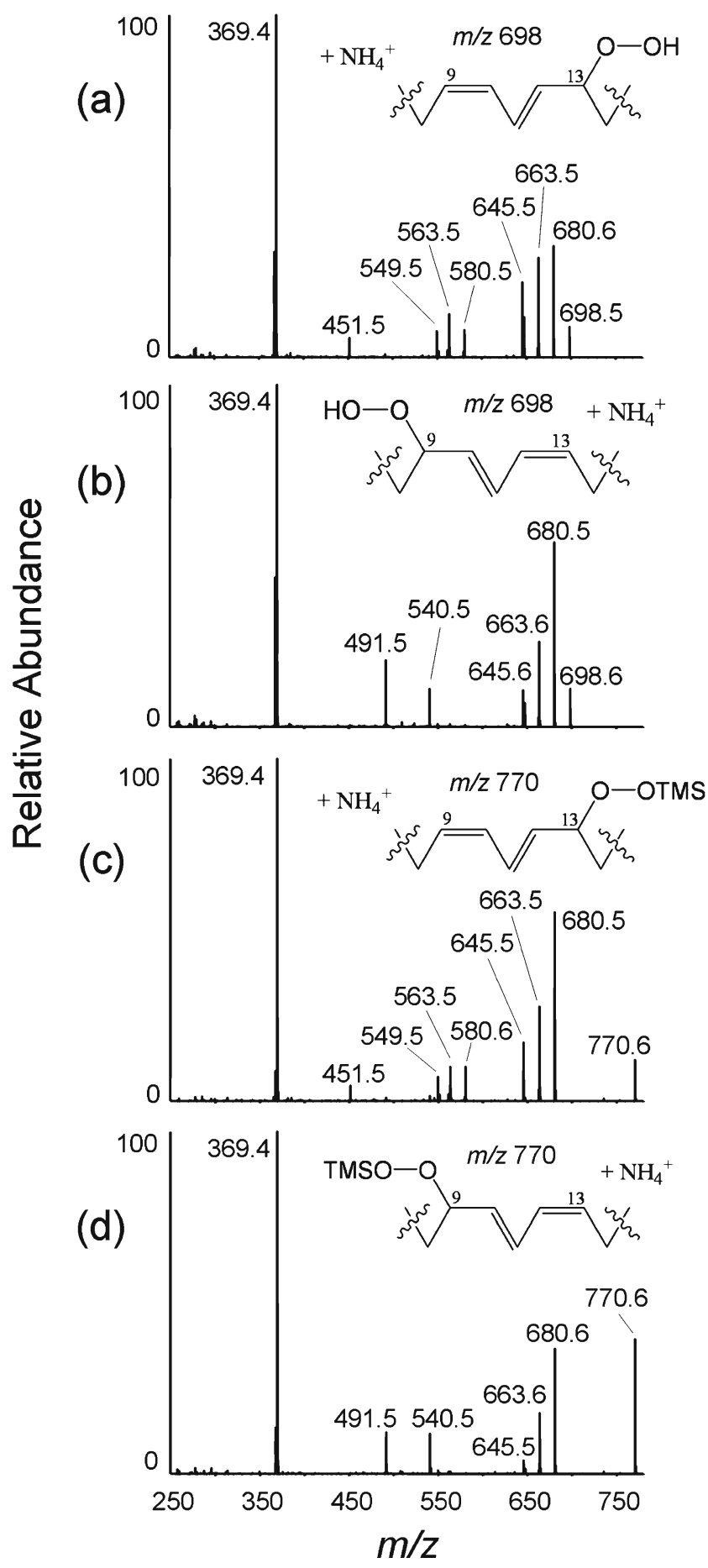

Figure 2. Collision induced fragmentation of the ammoniated molecular ions, $\left[\mathrm{M}+\mathrm{NH}_{4}\right]^{+}$, from (a) 13-HpODE-CE, (b) 9-HpODE-CE, (c) 13-HpODE-CE TMS ether (d) 9-HpODE-CE TMS ether. Structural details of the immediate hydroperoxide region are shown with the charging ammonium ion likely adducted at the ester moiety

To further elucidate the structures of CID product ions, deuterated-9 and 13-HpODE-CEs having deuterium atoms at carbon atoms 9, 10, 12, and 13 were synthesized and the ammoniated molecular ion species subjected to MS/MS.
Hereafter these CID data are referred to as $\mathrm{D}_{4}$-HpODE-CE product ion spectra. Major product ions from both labeled and unlabeled species along with observed mass shifts are summarized in Table 1. In other experiments, 9- or 13HpODE-CE (non-deuterated species) were introduced into the ion source after mixing with $\mathrm{D}_{2} \mathrm{O}$ and $\mathrm{ND}_{4} \mathrm{OAc}$ for several seconds to label readily exchangeable protons. In these experiments, the fully deuterated species, [ROOD + $\left.\mathrm{ND}_{4}\right]^{+} m / z$ 703, was isolated for fragmentation. These spectra revealed that a number of the CID product ions retained exchangeable deuterium atoms such as $\mathrm{m} / \mathrm{z} 542$ from 9-HpODE-CE and $\mathrm{m} / \mathrm{z} 583$ from 13-HpODE-CE. In another experiment, 9- and 13-HpODE-CEs were infused in a solution of ${ }^{15} \mathrm{NH}_{4} \mathrm{Cl}(3 \mathrm{mM})$ in $95: 5$ acetonitrile: $\mathrm{H}_{2} \mathrm{O}$ ( $\left.\mathrm{vol} / \mathrm{vol}\right)$ and subjected to MS/MS. Labeled product ions clearly revealed which ions retained the ammonium nitrogen atom, such as $m / z$ 681, 541, and 581 (Table 1). Interestingly, the major ion at $\mathrm{m} / \mathrm{z} 663$ did not retain any charging ammonium nitrogen atom, but as expected $m / z 680\left[\mathrm{M}+\mathrm{NH}_{4}-\mathrm{H}_{2} \mathrm{O}\right]^{+}$did. The full product ion mass spectra collected during these CID experiments are shown in Supplemental Figure 1.

To further examine the nature of these CE hydroperoxyspecific product ions, high-resolution accurate mass measurements were made of the major product ions. A mixture of 9- and 13-HpODE-CE was infused into an LTQ-FTICR MS, the ammoniated molecular ions were subjected to CID, and the product ions were detected at high resolution in the FTICR. The products ions were identical to those observed in other $\mathrm{MS}^{2}$ experiments, and accurate mass measurements were made. The measured $\mathrm{m} / \mathrm{z}$ values of the product ions were all within $1 \mathrm{ppm}$ of the calculated $\mathrm{m} / \mathrm{z}$ value for the hypothesized ion composition (Table 2).

\section{Discussion}

The fact that 9- and 13-HpODE-CE positional isomers produced such unique product ion spectra was an unexpected result. The isomer specific ions appeared between $\mathrm{m} / \mathrm{z}$ 450 and 580, suggesting a structure with an intact cholesteryl backbone and a portion of the acyl chain component. Such a structure would arise from cleavage along the acyl chain at or near the site of oxygen atom attachment. In contrast to these data, the CID spectra of 9and 13-oxo-ODE-CEs were identical to each other, and these compounds revealed no carbon-carbon bond cleavages of the acyl chain (Supplementary Figure 2A and B). The same was true for the pair of 9- and 13-hydroxyoctadecadienoic CEs (Supplementary Figure 2C and D). This behavior suggested that acyl chain cleavages observed in HpODECEs were dependent upon the hydroperoxide moiety consistent with the known tendency of hydroperoxides to undergo scission of the peroxide bond [21]. Previous studies suggested thermolysis of 9- and 13-HpODE TMS ethers was initiated by cleavage of the $\mathrm{O}-\mathrm{O}$ bond [11]. To further interrogate the structures of these product ions and the mechanisms of their genesis several experiments were 
Table 1. Summary of Major Product Ions Following Collisional Activation of Isotope Labeled and Unlabeled 9- and 13-HpODE-CE

\begin{tabular}{|c|c|c|c|c|}
\hline \multirow[b]{2}{*}{$\begin{array}{l}\text { 9-HpODE-CE } \\
{\left[\mathrm{M}+\mathrm{NH}_{4}\right]^{+}} \\
m / z 699\end{array}$} & \multirow[b]{2}{*}{$\begin{array}{l}\text { 13-HpODE-CE } \\
{\left[\mathrm{M}+\mathrm{NH}_{4}\right]^{+}} \\
m / z 699\end{array}$} & \multicolumn{3}{|c|}{$\mathrm{MS} / \mathrm{MS}$ of isotope labeled species } \\
\hline & & $\begin{array}{l}\text { D4-HpODE-CE } \\
{\left[\mathrm{M}+\mathrm{NH}_{4}\right]^{+}} \\
m / z 702\end{array}$ & $\begin{array}{l}\text { HpODE-CE }{ }^{\mathrm{b}} \\
{\left[\mathrm{M}+\mathrm{D}+\mathrm{ND}_{4}\right]^{+}} \\
m / z 703\end{array}$ & $\begin{array}{l}\text { HpODE-CE }{ }^{\mathrm{c}} \\
{\left[\mathrm{M}+{ }^{15} \mathrm{NH}_{4}\right]^{+}} \\
m / z 699\end{array}$ \\
\hline 680 & 680 & $684(+4)$ & $683(+3)$ & $681(+1)$ \\
\hline 663 & 663 & $667(+4)$ & $663(+0) / 664(+1)$ & $663(+0)$ \\
\hline 647 & 647 & $651(+4)$ & $647(+0)$ & $647(+0)$ \\
\hline 645 & 645 & $649(+4)$ & $645(+0)$ & $645(+0)$ \\
\hline 540 & - & $541(+1)$ & $542(+2)$ & $541(+1)$ \\
\hline 491 & - & $494(+3)$ & $491(+0)$ & $491(+0)$ \\
\hline- & 580 & $583(+3)$ & $583(+3)$ & $581(+1)$ \\
\hline - & 563 & $566(+3)$ & $563(+0)$ & $563(+0)$ \\
\hline - & 549 & $551(+3)$ & $549(+0)$ & $549(+0)$ \\
\hline - & 451 & $452(+1)$ & $451(+0)$ & $451(+0)$ \\
\hline
\end{tabular}

${ }^{\text {a Nominal }} \mathrm{m} / \mathrm{z}$ values of the individual ions

${ }^{\mathrm{b}}$ The number of deuterium atoms retained in each product ion is indicated in parentheses

${ }^{\mathrm{c}}$ The number of ${ }^{15} \mathrm{~N}$ atoms retained in each product ion is indicated in parentheses

carried out, each designed to elucidate specific features of product ion structures (Table 1, Supplemental Figure 1). Together, the results supported the hypothesis that scission of the relatively weak hydroperoxide $\mathrm{O}-\mathrm{O}$ bond was the initiating event in CID of HpODE CEs. The results also indicated that ammoniated HpODE CEs undergo complex dissociations, including the formation of covalent bonds between the analyte and the ammonium adduct itself.

The product ion at $\mathrm{m} / \mathrm{z} 680$, which was 18 u lower in mass than the ammonium adduct, was initially assumed to result from simple charge remote dehydration of the hydroperoxide. When the product ion spectrum from each of the carbon-chain deuterated hydroperoxides (Supplementary Figure $1 \mathrm{~A}$ and $\mathrm{B}$ ) was examined, no loss of any deuterium atoms for this product ion was observed (Table 1). This was in contrast to the negative ion CID behavior of arachidonate hydroperoxides, which loose the proton bearing the hydroperoxy group [14]. The $\mathrm{D}_{2} \mathrm{O} / \mathrm{ND}_{4}{ }^{+}$and ${ }^{15} \mathrm{NH}_{4}{ }^{+}$exchange experiments revealed that this ion retained the ammonium nitrogen and three exchangeable deuterium atoms. In comparison with the product ion mass spectra of the TMS derivatives, these exchangeable protons appeared to be derived exclusively from the ammonium adduct species, not the hydroperoxide proton. Homolytic cleavage of the hydroperoxide, accompanied by the expulsion of water (where the proton is from the ammonium adduct species) and alkoxyamine formation $\left[\mathrm{ROND}_{3}\right]^{+}$, was consistent with these data (Scheme 1a). The $m / z 580$ ion (in the 13-HpODE$\mathrm{CE}$ spectra) also retained the nitrogen atom and three exchangeable protons and was, therefore, hypothesized to be related to $m / z 680$. $\mathrm{MS}^{3}$ data $(\mathrm{m} / z$ 698 $\rightarrow 680 \rightarrow$ product ion scan) showed that $\mathrm{m} / \mathrm{z} 680$ was a precursor of $\mathrm{m} / \mathrm{z} 580$ (data not shown), likely occurring through neutral loss of the terminal portion of the acyl chain as an aldehyde (Scheme 1b). These experiments were carried out in the quadrupole linear ion trap hybrid mass spectrometer.

The ion at $\mathrm{m} / \mathrm{z} 663$ was initially thought to result from neutral loss of ammonia accompanied by dehydration of the hydroperoxide, and while the nitrogen atom was lost as supported by the high-resolution experiments (Table 2), the required deuterium atom was not lost in the $\mathrm{D}_{4}$-HpODE-CE spectra (Table 1). Curiously, for the 13-HpODE-CE with exchangeable protons labeled with deuterium atoms, the CID of $m / z 703$ yielded two product ions, $m / z 663$ and 664, suggesting two mechanisms producing isobaric products. Neutral loss of ammonia combined with heterolytic peroxide cleavage and $\mathrm{O}-\mathrm{O}$ bond formation would be consistent with one of these mechanisms (Scheme 1c). An alternate

Table 2. High resolution accurate mass measurements of molecular and product ions of 9- and 13-HpODE CE following collisional activation

\begin{tabular}{lccr}
\hline Proposed ion composition & Calculated $m / z\left(m_{a}\right)$ & Measured $m / z\left(m_{i}\right)^{\mathrm{a}}$ & $\Delta m_{i}(\mathrm{ppm})^{\mathrm{b}}$ \\
\hline $\mathrm{C}_{45} \mathrm{H}_{80} \mathrm{NO}_{4}{ }^{+}$ & 698.6082 & $698.6085 \pm 0.0006$ & 0.43 \\
$\mathrm{C}_{45} \mathrm{H}_{78} \mathrm{NO}_{3}^{+}$ & 680.5976 & $680.5978 \pm 0.0006$ & 0.35 \\
$\mathrm{C}_{45} \mathrm{H}_{75} \mathrm{O}_{3}^{+}$ & 663.5711 & $663.5713 \pm 0.0006$ & 0.23 \\
$\mathrm{C}_{45} \mathrm{H}_{73} \mathrm{O}_{2}^{+}$ & 645.5605 & $645.5606 \pm 0.0006$ & \\
$\mathrm{C}_{39} \mathrm{H}_{66} \mathrm{NO}_{2}^{+}$ & 580.5088 & $580.5087 \pm 0.0005$ & \\
$\mathrm{C}_{39} \mathrm{H}_{63} \mathrm{O}_{2}^{+}$ & 563.4823 & $563.4819 \pm 0.0004$ & -0.24 \\
$\mathrm{C}_{38} \mathrm{H}_{61} \mathrm{O}_{2}^{+}$ & 549.4666 & $549.4664 \pm 0.0004$ & -0.69 \\
$\mathrm{C}_{36} \mathrm{H}_{62} \mathrm{NO}_{2}^{+}$ & 540.4775 & $540.4773 \pm 0.0004$ & -0.42 \\
$\mathrm{C}_{36} \mathrm{H}_{59}^{+}$ & 491.4611 & $491.4607 \pm 0.0003$ & -0.44 \\
\hline
\end{tabular}

${ }^{\mathrm{a}}$ Mean \pm standard deviation for 20 measurements

${ }^{\mathrm{b}} \Delta m_{i}(\mathrm{ppm})=\frac{m_{i}-m_{a}}{m_{a}} \times 10^{6}$ 


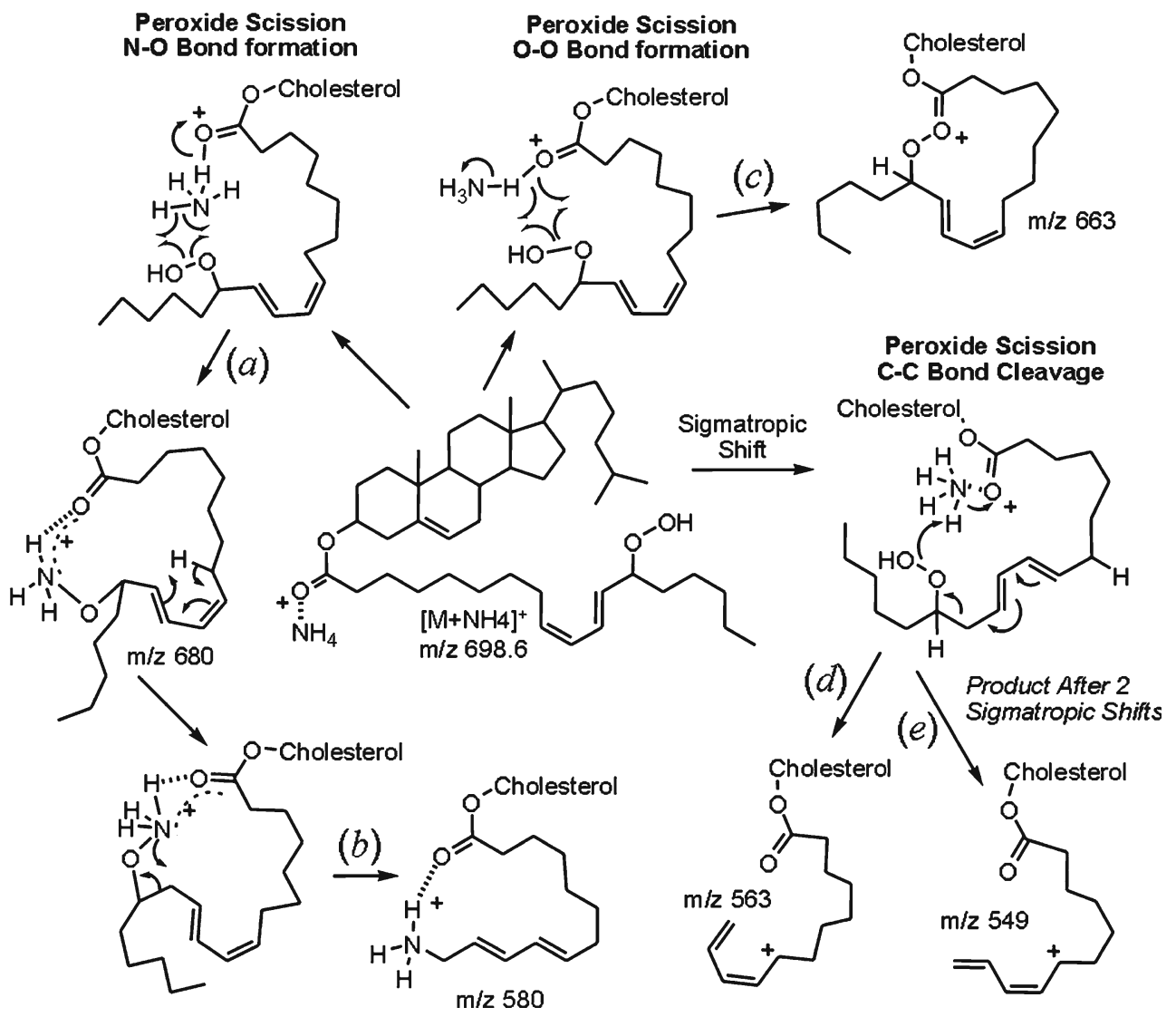

Scheme 1. Proposed mechanisms of collisional induced dissociation of ammoniated 13-HpODE CE, $\left[\mathrm{M}+\mathrm{NH}_{4}\right]^{+}$

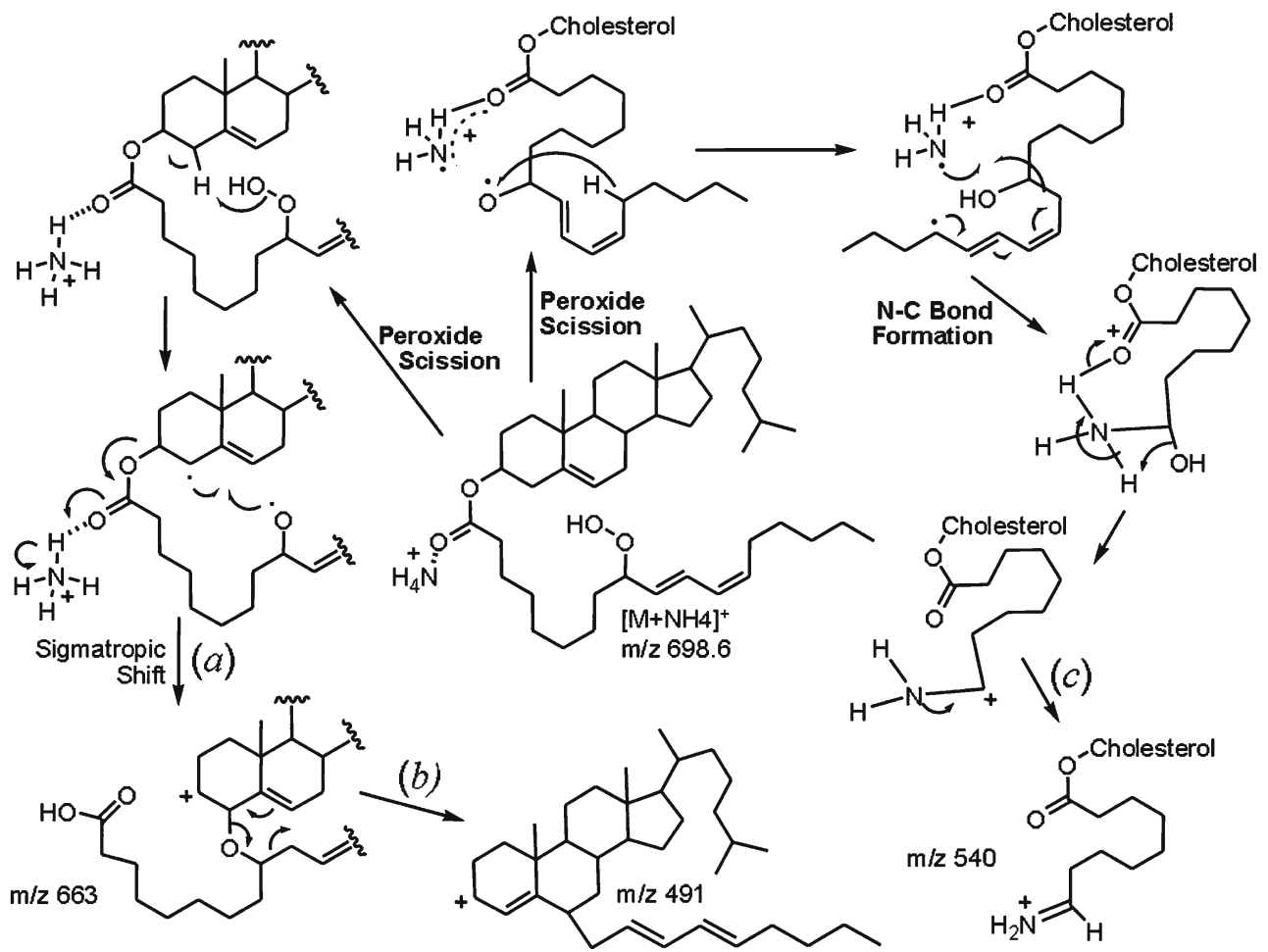

Scheme 2. Proposed mechanisms of collisional induced dissociation of ammoniated 9-HpODE-CE, $\left[\mathrm{M}+\mathrm{NH}_{4}\right]^{+}$ 
mechanism, which would fit for the ion retaining one exchangeable deuterium, would involve neutral loss of ammonia, breaking the ester bond that would yield a free carboxylic acid moiety, leaving a carbocation at carbon 3 of the cholesterol backbone. This is the same facile neutral loss reaction that produces the abundant $\mathrm{m} / \mathrm{z} 369$ (Figure 2a, b, c and d). This reaction would be the same as presented in Scheme $2 \mathrm{a}$ where homolytic cleavage of the peroxide bond and hydrogen abstraction from carbon-4 of cholesterol would drive the reaction with expulsion of water and ether bond formation. There is precedence for intramolecular attack of cholesterol-carbon 6 by peroxy radicals of HpODE-CE, as well as intermolecular attack by peroxy radicals between arachidonate hydroperoxides [22, 23]. $\mathrm{MS}^{3}$ experiments $(\mathrm{m} / z 698 \rightarrow 663 \rightarrow$ product ion scan) revealed that the fragment ion at $\mathrm{m} / \mathrm{z} 663$ (Scheme 2a) was a precursor of the product ion $\mathrm{m} / \mathrm{z} 491$ in the 9-HpODE-CE spectra (data not shown). An oxy-Cope rearrangement of the allylic ether [24] resulting in attachment of the terminal portion of the hydrocarbon chain to carbon 6 of the cholesterol core would be accompanied by the release of a protonated carboxylic acid-aldehyde $(172 \mathrm{u})$ and thereby explain this rather curious fragment ion (Scheme 2b). The high resolution data (Table 2) did reveal that the product ion $\mathrm{m} / \mathrm{z} 491$ contained only carbon and hydrogen atoms and no heteroatoms from the starting hydroperoxyl cholesterol ester ammonium adduct precursor ion. From the exact mass difference of the precursor $(\mathrm{m} / \mathrm{z} 663)$ and product ion $(\mathrm{m} / \mathrm{z}$ 491), the elemental composition of the suspected protonated carboxylic acid-aldehyde loss would be $\mathrm{C}_{9} \mathrm{H}_{16} \mathrm{O}_{3}$.

The ions at $\mathrm{m} / \mathrm{z} 563$ and 549 in the 13-HpODE-CE spectra retain three and two deuterium atoms in the $\mathrm{D}_{4}$ HpODE-CE spectra, respectively, but neither ion retained the nitrogen atom or exchangeable deuterium atoms. These data were consistent neutral loss of water and ammonium accompanied by carbon-carbon bond cleavage proximal to the substituted carbon atom, losing a neutral aldehyde (as illustrated in Scheme 1d) or losing an epoxide (pathway Scheme 1e) where both losses would leave a resonance stabilized carbocation $(\mathrm{m} / \mathrm{z} 563$ and 549). The site of cleavage and the length of the remaining acyl component depended on the rearrangement of double bonds prior to fragmentation. Consistent with this mechanism was that similar fragments were not observed in the 9-HpODE-CE spectra, as the ionized species in that case would be the terminal end of the acyl component

The ion at $m / z 540$ in the 9-HpODE-CE retained one deuterium in the $\mathrm{D}_{4}$-HpODE-CE spectra as well as the nitrogen atom in the ${ }^{15} \mathrm{NH}_{4}$ product ion spectra and highresolution experiments and, curiously, retained two exchangeable deuterium atoms. A structure that included a terminal imine would account for the retention of the nitrogen and two exchangeable deuterium atoms. The single backbone deuterium attached to this structure most likely resided at carbon-9, where the terminal acyl portion was lost. This complex ion structure could be rationalized in the context of initial peroxide scission leading to the expulsion of water and the formation of the first carbon nitrogen bond when the terminal acyl portion is lost as a neutral hydrocarbon. The second peroxide oxygen is then lost as water and the protonated imine is formed (Scheme 2c).

\section{Conclusions}

Hydroperoxides of linoleate cholesteryl esters formed abundant ammoniated molecular ions during electrospray ionization in the positive ion mode. Little dehydration of the molecular species occurred during ionization in contrast to electrospray ionization of free hydroperoxide fatty acid carboxylate anions, which undergo extensive dehydration in the ion source. Collision induced dissociation of each ammoniated positional isomer (9- and 13-HpODE-CE) resulted in distinct product ion spectra. Ions unique to the positional isomer result from carbon-carbon bond cleavage along the acyl component. All acyl chain fragmentation events appeared to be initiated by scission of the hydroperoxide moiety and further directed by the relative positions of the hydroperoxide and of double bonds along the acyl chain.

\section{Acknowledgments}

The authors acknowledge support in part for this work by the LIPIDMAPS Large Scale Collaborative grant (GM069338) from the General Medical Sciences Institute of the National Institutes of Health. They acknowledge the University of Colorado Denver, Proteomic Mass Spectrometry Shared Resource.

\section{References}

1. Murphy, R.C., Gijón, M.A.: Biosynthesis and metabolism of leukotrienes. Biochem. J. 405, 379-395 (2007)

2. Smith, W.L.: Nutritionally essential fatty acids and biologically indispensable cyclooxygenases. Trends Biochem. Sci. 33, 27-37 (2008)

3. Spector, A.A.: Arachidonic acid cytochrome P450 epoxygenase pathway. J. Lipid Res. 50, S52-S56 (2009)

4. Pace-Asciak, C.R., Asotra, S.: Biosynthesis, catabolism, and biological properties of HPETEs, hydroperoxide derivatives of arachidonic acid. Free Radic. Biol. Med. 7, 409-433 (1989)

5. Porter, N.A., Caldwell, S.E., Mills, K.A.: Mechanisms of free radical oxidation of unsaturated lipids. Lipids 30, 277-290 (1995)

6. Hazen, S.L.: Oxidized phospholipids as endogenous pattern recognition ligands in innate immunity. J. Biol. Chem. 283, 15527-15531 (2008)

7. Harkewicz, R., Hartvigsen, K., Almazan, F., Dennis, E.A., Witztum, J. L., Miller, Y.I.: Cholesteryl ester hydroperoxides are biologically active components of minimally oxidized low density lipoprotein. J. Biol. Chem. 283, 10241-10251 (2008)

8. Heinecke, J.W.: Oxidants and antioxidants in the pathogenesis of atherosclerosis: implications for the oxidized low density lipoprotein hypothesis. Atherosclerosis 141, 1-15 (1998)

9. Gella, A., Durany, N.: Oxidative stress in alzheimer disease. Cell Adh. Migr. 3, 88-93 (2009)

10. Handelman, G.J.: High-performance liquid chromatography analysis of cholesterol linoleate hydroperoxide in oxidized low density lipoproteins: calibration by conjugated diene internal standard. Meth. Enzymol. 300, 4350 (1999)

11. Grechkin, A.N., Mukhtarova, L.S., Hamberg, M.: Thermal conversions of trimethylsilyl peroxides of linoleic and linolenic acids. Chem. Phys. Lipids 138, 93-101 (2005) 
12. Kawai, Y., Miyoshi, M., Moon, J., Terao, J.: Detection of cholesteryl ester hydroperoxide isomers using gas chromatography-mass spectrometry combined with thin-layer chromatography blotting. Anal. Biochem. 360, 130-137 (2007)

13. Hall, L.M., Murphy, R.C.: Electrospray mass spectrometric analysis of 5-hydroperoxy and 5-hydroxyeicosatetraenoic acids generated by lipid peroxidation of red blood cell ghost phospholipids. J. Am. Soc. Mass Spectrom. 9, 527-532 (1998)

14. MacMillan, D.K., Murphy, R.C.: Analysis of lipid hydroperoxides and long-chain conjugated keto acids by negative ion electrospray mass spectrometry. J. Am. Soc. Mass Spectrom. 6, 1190-1201 (1995)

15. Murphy, R.C., Barkley, R.M., Zemski Berry, K., Hankin, J., Harrison, K., Johnson, C., Krank, J., McAnoy, A., Uhlson, C., Zarini, S.: Electrospray ionization and tandem mass spectrometry of eicosanoids. Anal. Biochem. 346, 1-42 (2005)

16. Leitinger, N.: Cholesteryl ester oxidation products in atherosclerosis. Mol. Aspects Med. 24, 239-250 (2003)

17. Hutchins, P.M., Barkley, R.M., Murphy, R.C.: Separation of cellular nonpolar neutral lipids by normal-phase chromatography and analysis by electrospray ionization mass spectrometry. J. Lipid Res. 49, 804-813 (2008)

18. Zemski Berry, K.A., Turner, W.W., Van Nieuwenhze, M.S., Murphy, R. C.: Stable isotope labeled 4-(dimethylamino)benzoic acid derivatives of glycerophosphoethanolamine lipids. Anal. Chem. 81, 6633-6640 (2009)
19. Quehenberger, O., Armando, A.M., Brown, A.H., Milne, S.B., Myers, D.S., Merrill, A.H., Bandyopadhyay, S., Jones, K.N., Kelly, S., Shaner, R.L., Sullards, C.M., Wang, E., Murphy, R.C., Leiker, T.J., Raetz, C.R., Guan, Z., Laird, G.M., Six, D.A., Russell, D.W., McDonald, J.G., Subramaniam, S., Fahy, E., Dennis, E.A.: Lipidomics reveals a remarkable diversity of lipids in human plasma. J. Lipid Res. 51, 3299-3305 (2010)

20. Duffin, K., Obukowicz, M., Raz, A., Shieh, J.J.: Electrospray/tandem mass spectrometry for quantitative analysis of lipid remodeling in essential fatty acid deficient mice. Anal. Biochem. 279, 179-188 (2000)

21. Blée, E., Wilcox, A.L., Marnett, L.J., Schuber, F.: Mechanism of reaction of fatty acid hydroperoxides with soybean peroxygenase. $J$. Biol. Chem. 268, 1708-1715 (1993)

22. Spiteller, G.: Linoleic acid peroxidation - the dominant lipid peroxidation process in low density lipoprotein — and its relationship to chronic diseases. Chem. Phys. Lipids 95, 105-162 (1998)

23. Schneider, C., Boeglin, W.E., Yin, H., Porter, N.A., Brash, A.R.: Intermolecular peroxyl radical reactions during autoxidation of hydroxy and hydroperoxy arachidonic acids generate a novel series of epoxidized products. Chem. Res. Toxicol. 21, 895-903 (2008)

24. Wheelan, P., Zirrolli, J.A., Murphy, R.C.: Low-energy fast atom bombardment tandem mass spectrometry of monohydroxy substituted unsaturated fatty acids. Biol. Mass Spectrom. 22, 465-473 (1993) 